\title{
Prevalence of suspected developmental coordination disorder and associated factors in Spanish classrooms
}

\author{
Laura Delgado-Lobete, Sergio Santos-del-Riego, Sonia Pértega-Díaz, Rebeca Montes- \\ Montes
}

University of A Coruña, Faculty of Health Sciences, Campus de Oza S/N, A Coruña, Spain

\begin{abstract}
Background. Developmental Coordination Disorder (DCD) is a multifactorial, neurodevelopmental motor disorder that severely affects the activities of a child's daily life and classroom performance. The aim of this study was to determine the prevalence of suspected DCD in a sample of Spanish schoolchildren and its association with sociodemographic factors.

Methods. We conducted a cross-sectional study including a random sample of 460 children attending mainstream schools in northwest Spain in 2017. A Developmental Coordination Disorder Questionnaire-European Spanish was used to evaluate suspected DCD prevalence. We performed multivariate logistic and linear regression analysis to determine the socio-demographic variables associated with suspected DCD and problematic motor coordination performance.

Results. The prevalence of suspected DCD was $12.2 \%$. According to the multivariate analysis, DCD symptoms were significantly associated with males $(\mathrm{OR}=3.0)$, ages above 10 years old $(\mathrm{OR}=5.0)$ and low participation in out-ofschool physical activities $(\mathrm{OR}=2.3)$. Preterm birth children were twice as likely to show suspected $\mathrm{DCD}$, although this association was not statistically significant $(\mathrm{OR}=2.1)$.

Conclusions. A high percentage of Spanish schoolchildren are at risk for developing DCD. There is a strong connection between suspected DCD and socio-demographic factors. Protocols aimed to detect DCD and intervention programmes in classrooms designed to promote motor coordination skills need to take these factors into consideration.
\end{abstract}

\section{Keywords}

Developmental coordination disorder; Child development; Psychomotor performance; Epidemiology; Prevalence 


\section{What this paper adds?}

This is the first study to investigate the epidemiology of suspected Developmental Coordination Disorder (DCD) in a large sample of Spanish schoolchildren aged 6-12 years old. Approximately three children in a general Spanish classroom are at risk for DCD. Boys are three times more likely to show coordination difficulties. Age, premature birth and low participation in out-of-school physical activities are also associated with DCD symptoms. These findings indicate that factors associated with motor coordination difficulties in schoolchildren span across neurological and socio-demographic domains, and should be addressed during early development to promote prompt interventions. Schools offer the best setting to detect learning difficulties due to motor coordination issues, and teachers and health care practitioners working in schools can use this study to design specific strategies to promote full participation according to these children's needs and characteristics.

\section{Introduction}

Developmental Coordination Disorder (DCD) is a motor neurodevelopmental disorder characterized by a significant delay in the acquisition and execution of coordinated motor skills as expected for the child's chronological age and opportunities for learning (American Psychiatry Association, 2013; Blank, Smits-Engelsman, Polatajko, \& Wilson, 2012). DCD affects approximately 5-19\% of school-aged children, varying according to the diagnosis criteria and country (Blank et al., 2012; Beltrame et al., 2017; Faebo Larsen, Hvas Mortensen, Martinussen, \& Nybo Andersen, 2013; Lingam, Hunt, Golding, Jongmans, \& Emond, 2009; Pulzi \& Rodrigues, 2015; Santos \& Vieira, 2013; Valentini et al., 2012). The underlying motor and behavioural difficulties of DCD are chronic and severely limit activities of daily living, including educational achievement and classroom performance (Magalhães, Cardoso, \& Missiuna, 2011). Children with DCD show great difficulties in social and academic tasks and in scholastic achievements (Blank et al., 2012). De Milander, Coetzee, and Venter (2016) found that motor coordination difficulties were associated with impaired reasoning, numerical skills, pattern repeating, fine motor skills and memory outcomes in children aged 5-8 and concluded that children with DCD experience more learning-related problems than children without DCD. Previous research has shown that this disorder significantly interferes with classroom tasks and demands like reading and writing, maintaining attention and numerical-mathematical comprehension (Gomez et al., 2015; Huau, Velay, \& Jover, 2015; Prunty, Barnett, Wilmut, \& Plumb, 2016).

According to the American Psychiatric Association's Diagnostic and Statistical Manual of Mental Disorders (DSM-5), DCD is defined by the following four criteria: A) Motor coordination performance is substantially below that expected given the person's chronological age and opportunity for skill learning and use; B) This motor coordination deficit significantly and persistently interferes with typical chronological age activities of daily living, including school performance; C) Onset of symptoms is in the early developmental period; and D) The motor coordination deficit is not better explained by intellectual disability or visual impairment and cannot be attributable to a neurological condition affecting movement (American Psychiatry Association, 2013). The European Academy of Childhood Disability recommends using the Movement Assessment Battery for Children-Second Edition (MABC-2) and the Developmental Coordination Disorder Questionnaire (DCDQ) as assessment tools to evaluate criteria A and B, respectively (Blank et al., 2012).

Research shows that the underlying mechanisms of DCD include both internal and external factors (Blank et al., 2012). It has been proposed that neurological, behavioural and contextual variables influence movement performance through a dynamic systems approach (Haywood, Roberton, \& Getchell, 2012; Shumway-Cook \& Woollacott, 2010). Recent meta-analysis studies have found that children with DCD show anomalies in sensorimotor processes, attention and task-oriented process neurobiology that could contribute to some of their underlying problems in anticipatory motor planning (Fuelscher et al., 2018; Gomez \& Sirigu, 2015; Wilson et al., 2017). 
To understand the underlying factors of DCD, it is necessary to know how socio-demographics affect motor coordination development. Although some variables are commonly associated with DCD, there is not a consensus regarding this topic. The prevalence of DCD is found to be higher in boys (Beltrame et al., 2017; Rivard, Missiuna, McCauley, \& Cairney, 2014), while some studies have not found a significant association with the sex of the children (Lingam et al., 2009; Silva \& Beltrame, 2013). This fact could be influenced by the tool used to assess DCD, since boys tend to score better on gross motor and aiming and catching tasks, but girls usually perform fine motor activities better than boys (AmadorRuiz et al., 2018; Beltrame et al., 2017; Delgado-Lobete \& Montes-Montes, 2017). Low gestational age at birth has been noted as a risk factor for developing DCD, especially in young children, due to cognitive and behavioural outcomes (Faebo Larsen et al., 2013; Kieviet, Piek, Aarnoudse-Moens, \& Oosterlaan, 2009; Lingam et al., 2009). Low socio-economic family status has been previously associated with DCD, as well as poor motor coordination. Children coming from disadvantaged families may have increased difficulties with accessing learning opportunities or resources, which may be a risk factor for developing DCD (Barba, Luiz, Pinheiro, \& Lourenço, 2017; Lingam et al., 2009; Valentini, Clark, \& Whitall, 2015). Another well-documented factor associated with DCD is low participation in out-of-school physical activities. Children with DCD tend to engage less in physical activities and sports than children without motor difficulties (Cermak et al., 2015; Magalhães et al., 2011). While restrictions in physical activities limits the number of opportunities to practice and improve motor skills, children with DCD show low self-perception about their physical competence, which may explain their reduced participation (Batey et al., 2014; Schoemaker \& Smits-Engelsman, 2015). The Spanish national curriculum for elementary education only requires two weekly hours of physical education and previous research has suggested that this allotted time may not be in compliance with the World Health Organization guidelines on physical activity for school-aged children (Calahorro Cañada, Torres-Luque, López-Fernández, \& Álvarez Carnedo, 2014; Pons \& Arufe, 2015). For this reason, it is important to assess participation in out-ofschool physical activity when addressing a DCD evaluation.

Due to the adverse impact that DCD can have on the daily life activities and classroom performance of schoolchildren, it is necessary to know the epidemiology of this disorder in Spanish, general education classrooms to provide guidance and early identification programmes to teachers and schools. Considering DCD often goes undiagnosed, it is important that teachers and health practitioners working in schools can detect children that struggle because of learning difficulties, due to motor coordination issues, and the primary socio-demographic factors associated with DCD in schoolchildren (Missiuna et al., 2008). Early detection of motor difficulties could prompt effective control of these children to prevent worse, secondary consequences, help teachers design an Individualized Education Plan that accommodates the children's strengths and needs or promote access to targeted materials and resources (Missiuna, Moll, King, King, \& Law, 2006).

In this context, Spanish classrooms may have a difficult time detecting and assessing this disorder because of several factors, such as the lack of information and underdiagnosis of DCD in the Spanish population (Amador-Ruiz et al., 2018; Carballal Mariño et al., 2018). To our knowledge, only one study has explored DCD in Spanish children, but it used a small study sample to evaluate criterion A, did not include children older than six years old, and did not consider gestational age at birth or participation in out-of-school physical activities (Amador-Ruiz et al., 2018). Considering the existing problems for the diagnosis of DCD in children under 5 years of age and the lack of stability of DCD at early ages (Blank et al., 2012), their findings may differ in older Spanish children. Additionally, the absence of health care practitioners who are familiar with DCD and could assist in detecting children with motor coordination difficulties in mainstream schools, like occupational or physical therapists, adds to the difficulty of detecting and assessing DCD in Spanish classrooms. For this reason, knowing the suspected prevalence of DCD and associated socio-demographic factors in Spanish schoolchildren can provide useful information to guide specific detection strategies and intervention programmes. International researchers could compare the prevalence of suspected DCD in Spanish schoolchildren in relation to other regions due to socio-demographic or cultural differences. Additionally, knowing how interconnected sociodemographic factors associate with motor coordination difficulties can contribute to the theoretical background explaining the underlying factors involving DCD. 
To date, few studies have explored the underlying associations between DCD, motor coordination dimensions and socio-demographic factors using multivariate regression models, and no study has established suspected DCD prevalence in a large sample of Spanish school-aged children. It is important to explore how socio-demographic factors individually associated with DCD interconnect in schoolchildren. Teachers and health care practitioners working in schools can easily obtain children's sex, age, participation in out-of-school physical activity, family socioeconomic background and preterm status. Knowing how these factors associate with DCD could help teachers and health practitioners in Spain, and other regions, to design strategies for the early detection of children more vulnerable to the development of motor coordination difficulties that could impact academic achievement, social performance and behaviour in classrooms.

The objective of this study was to estimate the prevalence of suspected DCD in a large sample of Spanish children aged 6-12 years, attending mainstream schools, and to determine the associations between suspected DCD, problematic motor coordination ability in different areas and socio-demographic factors.

\section{Methods}

\subsection{Procedure and participants}

We conducted a cross-sectional study in A Coruna, northwest Spain. According to the Galician Institute of Statistics, 14,466 schoolchildren between six and twelve years of age were eligible for the study (Instituto Gallego de Estadística, 2017). With 95\% confidence limit, an expected DCD prevalence of $15 \%$ and a precision of .035 , the required sample size was 432 as calculated by EPIDAT 3.1.

Eight general education schools, randomly selected, were invited to participate in the study, of which six agreed to collaborate. A dossier including the Developmental Coordination Disorder QuestionnaireEuropean Spanish (DCDQ-ES) and a socio-demographic questionnaire was issued to 1002 randomly selected parents of children from first to sixth grade (aged 6 to 12 years). Parents were also asked whether their children had a clinical diagnosis of any developmental disorder or learning difficulties. The dossiers were anonymously answered at home and then returned to schools and then collected by the researchers. Only fully completed DCDQ-ES were considered valid and therefore included in the study.

Ethical approval for this study was obtained from the Autonomic Research Ethics of Galicia Committee (code 2017/166). All participants consented to take part anonymously and confidentially.

\subsection{Assessment measures}

Children were defined as having suspected DCD using the European Spanish cross-cultural adaptation of the DCDQ (to be published). The DCDQ is a parent-questionnaire consisting of 15 items that asses three coordination factors when performing activities of daily living: control during movement, fine motor/handwriting and general coordination. Each item scores from 1 to 5, where lower scores are indicative of coordination difficulties. As recommended by Wilson et al. (2009), children were defined as having suspected DCD if they had a total score of 46 or below (ages 6 years to 7 years 11 months), 55 or below (ages 8 years to 9 years 11 months) or 57 or below (ages 10 years to 12 years 11 months). The DCDQ has shown good psychometric properties (Cronbach $\alpha=0.94$; overall sensitivity $=85 \%$; overall specificity $=71 \%$ ) (Wilson et al., 2009). The DCDQ is a well-validated tool, useful as a first step diagnostic assessment, especially to support and operationalize criterion B (Blank et al., 2012). 
Covariables included were gender, age, gestational age at birth, participation in out-of-school physical activities and educational and occupational family levels. The parents' educational level was measured using the International Standard Classification of Education (UNESCO Institute for Statisfics, 2012), while occupational level was assessed with the occupational classification proposed by the Spanish Society of Epidemiology and the Spanish Society of Family and Community Medicine (DomingoSalvany, Regidor, Alonso, \& Alvarez-Dardet, 2000).

\subsection{Data analysis/calculation}

We conducted a descriptive analysis calculating percentages with their 95\% confidence interval (CI) for categorical variables (e.g., suspected DCD, sex, family educational level, etc.), and means and standard deviation (SD) for numerical variables (e.g., age, motor coordination factors). Student t test, ANOVA analyses and Chi Square were used to determine the associations between suspected DCD, problematic ability in the coordination factors and socio-demographic variables. Student tests were conducted for assessing associations between problematic ability in motor coordination factors and sex, gestational age at birth, out-of-school physical activity and father, mother and family educational and occupational levels, while ANOVA analyses were conducted for assessing associations between motor coordination factors and age groups. Chi Square tests $\left(\mathrm{X}^{2}\right)$ were used to explore the associations between suspected DCD and socio-demographic variables (e.g., sex, age groups, gestational age at birth, etc.). Finally, logistic and linear regression models were used to determine which variables were associated with suspected DCD and problematic coordination performance. All analyses were performed using SPSS v. 20 and EPIDAT v. 3.1. A minimum alpha level of 0.05 was set for all statistical tests.

\section{Results}

The sample for this study was comprised of 460 schoolchildren ( $45.9 \%$ rate of valid response) $\left(\mathrm{M}_{\mathrm{age}}=\right.$ $8.66, \mathrm{SD}= \pm 1.79$; girls $=53.0 \%$ ). Only one child had a parent reported, clinical diagnosis of Attention Deficit and Hyperactivity Disorder (ADHD, 0.2\%, 95\% CI $=0.0-1.2$ ) and was included in the study. Socio-demographic characteristics are shown in Table 1. Fifty-six children were identified as having suspected DCD $(12.2 \%, 95 \% \mathrm{CI}=9.1 \%-15.3 \%)$. Boys were more likely to show suspected DCD than girls in all age groups as shown in Fig. 1. 
Table 1. Socio-demographic characteristics of participants.

Participants $\quad$ Mean \pm SD or $\%(95 \% \mathrm{CI})$

Age

6-7 years

8-9 years

10 and more years

Sex (girls)

Developmental disorders or learning difficulties

Full term birth

Out-of-school physical activity ( $\geq 3 \mathrm{~h} /$ week)

Father educational level (third level studies)

Mother educational level (third level studies)

Family educational level (third level studies)

Father occupational level (occupation corresponding to university studies)

Mother occupational level (occupation corresponding to university studies)

Family occupational level (occupation corresponding to university studies)
$8.66 \pm 1.8$

$31.1(26.8-35.4)$

$33.5(29.1-37.9)$

$35.4(31.0-39.9)$

$53.0(48.4-57.7)$

$0.2(0.0-1.2)$

$83.6(79.8-87.4)$

$49.1(44.4-53.8)$

$70.7(66.3-75.1)$

$80.8(77.0-84.5)$

$84.9(81.6-88.3)$

$25.6(21.3-29.9)$

$27.6(23.3-31.78)$

$35.1(30.6-39.6)$

$\mathrm{SD}=$ standard deviation $; 95 \% \mathrm{CI}=95 \%$ confidence interval.

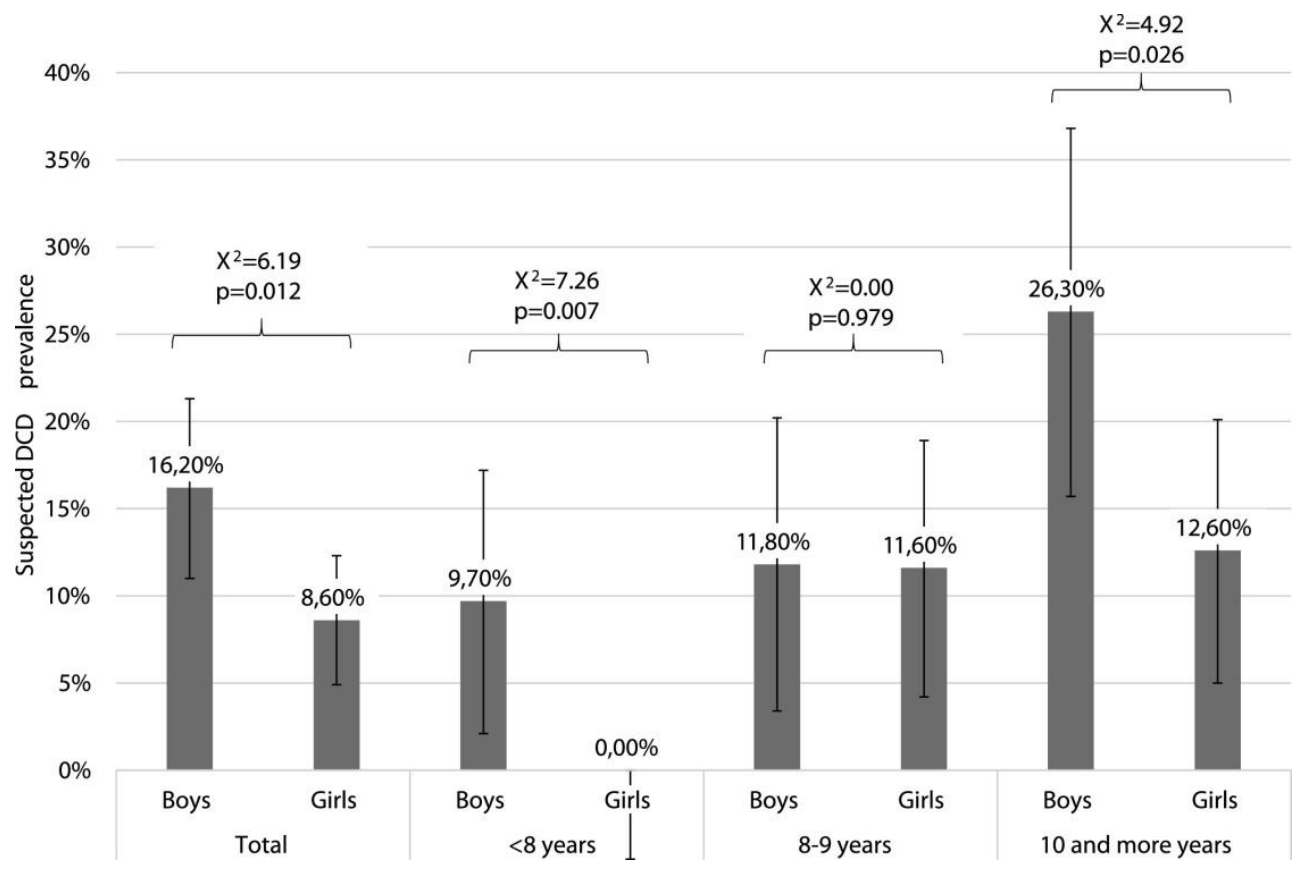

Fig. 1. Suspected DCD prevalence differences according to sex and age groups. $X^{2}=$ chi square value; $p=p$ value. 
Suspected DCD was associated with being male, age, preterm birth, low out-of-school physical activity and family educational level (Table 2). Prevalence of suspected DCD was higher among boys $\left(16.2 \%\right.$ vs $\left.8.6 \%, \mathrm{X}^{2}=6.19, \mathrm{df}=1\right)$, children older than 10 years of age $\left(19.0 \%\right.$ vs $8.4 \%, \mathrm{X}^{2}=14.26$, $\mathrm{df}=2)$, preterm children $(18.8 \%$ vs $9.8 \%,=4.27, \mathrm{df}=1)$, children who enjoy less than three hours per week of out-of-school physical activities $\left(15.3 \%\right.$ vs $\left.8.1 \%, \mathrm{X}^{2}=5.52, \mathrm{df}=1\right)$ and children with a low family educational level $\left(20.3 \%\right.$ vs $\left.10.5 \%, X^{2}=5.27, \mathrm{df}=1\right)$ regardless of family occupational level.

Table 2. Association between socio-demographic factors and suspected DCD using Chi-square test.

\begin{tabular}{|c|c|c|c|c|c|c|}
\hline & No DCD N (\%) & Suspected DCD N (\%) & $\mathrm{X}^{2}$ & $\mathrm{p}$ & OR & $95 \% \mathrm{CI}$ \\
\hline Sex & & & 6.19 & 0.013 & 2.05 & $1.2-3.7$ \\
\hline Boys & $181(83.8)$ & $35(16.2)$ & & & & \\
\hline Girls & $223(91.4)$ & $21(8.6)$ & & & & \\
\hline Age & & & 14.26 & 0.001 & & \\
\hline $6-7$ years & $136(95.1)$ & $7(4.9)$ & & & 1 & - \\
\hline $8-9$ years & $136(88.3)$ & $18(11.7)$ & & & 0.22 & $0.1-0.5$ \\
\hline 10 and more years & $132(81.0)$ & $31(19.0)$ & & & 0.57 & $0.3-1.1$ \\
\hline Gestational age at birth & & & 4.27 & 0.039 & 2.12 & $1.0-4.4$ \\
\hline Preterm & $52(81.2)$ & $12(18.8)$ & & & & \\
\hline Full term & $294(90.2)$ & $32(9.8)$ & & & & \\
\hline Out-of-school physical activity & & & 5.52 & 0.019 & 2.03 & $1.1-3.7$ \\
\hline$<3 \mathrm{~h} /$ week & $194(84.7)$ & $35(15.3)$ & & & & \\
\hline$\geq 3 \mathrm{~h} /$ week & 203 (91.9) & $18(8.1)$ & & & & \\
\hline Father educational level & & & 2.40 & 0.122 & 1.60 & $0.9-2.9$ \\
\hline First or second level studies & $106(84.1)$ & $20(15.9)$ & & & & \\
\hline Third level studies & $272(89.5)$ & $32(10.5)$ & & & & \\
\hline Mather educational level & & & 3.18 & 0.075 & 1.80 & $0.9-3.4$ \\
\hline First or second level studies & $71(82.6)$ & $15(17.4)$ & & & & \\
\hline Third level studies & $323(89.5)$ & $38(10.5)$ & & & & \\
\hline Family educational level & & & 5.27 & 0.022 & 2.16 & $1.1-4.2$ \\
\hline First or second level studies & $55(79.7)$ & $14(20.3)$ & & & & \\
\hline Third level studies & $348(89.5)$ & $41(10.5)$ & & & & \\
\hline Father occupational level & & & 2.13 & 0.144 & 1.74 & $0.8-3.7$ \\
\hline Occupation not corresponding to university studies & $274(86.4)$ & $43(13.6)$ & & & & \\
\hline Occupation corresponding to university studies & $100(91.7)$ & $9(8.3)$ & & & & \\
\hline Mother occupational level & & & 0.04 & 0.840 & 1.07 & $0.6-2.1$ \\
\hline Occupation not corresponding to university studies & $284(87.9)$ & $39(12.1)$ & & & & \\
\hline Occupation corresponding to university studies & $109(88.6)$ & $14(11.4)$ & & & & \\
\hline Family occupational level & & & 0.80 & 0.371 & 1.33 & $0.7-2.5$ \\
\hline Occupation not corresponding to university studies & $258(87.2)$ & $38(12.8)$ & & & & \\
\hline Occupation corresponding to university studies & $144(90.0)$ & $16(10.0$ & & & & \\
\hline
\end{tabular}

$X^{2}=$ chi square value; $p=p$ value; $O R=$ odds ratio $; 95 \% C I=95 \%$ confidence interval. 
Logistic regression analysis outcomes are presented in Table 3. We found that being male $[\mathrm{OR}=3.0$, 95\% CI (1.5-6.0)], being above 10 years of age [OR $=5.0,95 \%$ CI $(1.9-13.1)]$ and having a low participation in out-of-school physical activities $[\mathrm{OR}=2.3,95 \%$ CI $(1.1-4.7)]$ were statistically associated with suspected DCD. Family educational level and preterm birth were not statistically associated factors according to this analysis, although preterm children were twice as likely to show suspected DCD compared to children who were not preterm $[\mathrm{OR}=2.1,95 \% \mathrm{CI}(1.0-4.6)]$.

Table 3. Logistic multivariate analysis to identify socio-demographic factors associated with suspected DCD

\begin{tabular}{|c|c|c|c|c|}
\hline Variable & $\mathrm{B}$ & SE & $\mathrm{p}$ & OR $(95 \% \mathrm{CI})$ \\
\hline Sex (boys) & 1.081 & 0.358 & 0.003 & $3.0(1.5 ; 6.0)$ \\
\hline Age & & & 0.004 & \\
\hline $6-7$ years & & & & 1 \\
\hline 8 y 9 years & 0.932 & 0.518 & 0.072 & $2.5(0.9 ; 7.0)$ \\
\hline 10 and more years & 1.603 & 0.496 & 0.001 & $5.0(1.9 ; 13.1)$ \\
\hline Preterm birth (<37 weeks) & 0.745 & 0.397 & 0.06 & $2.1(1.0 ; 4.6)$ \\
\hline Out-of-school physical activity ( $<3 \mathrm{~h} /$ week) & 0.838 & 0.364 & 0.021 & $2.3(1.1 ; 4.7)$ \\
\hline Family educational level (first or second level studies) & 0.450 & 0.426 & 0.291 & $1.6(0.7 ; 3.6)$ \\
\hline
\end{tabular}

$\mathrm{B}=\mathrm{B}$ coefficient value; $\mathrm{SE}=$ standard error; $\mathrm{OR}=$ odds ratio; $\mathrm{p}=\mathrm{p}$ value; $95 \% \mathrm{CI}=95 \%$ confidence interval.

Regarding the socio-demographic factors associated with coordination factors and the total DCDQ-ES score, girls, children who participated in three or more hours in out-of-school physical activities and children whose families had high educational and occupational levels scored significantly higher in DCDQ-ES, showing fewer problematic levels of ability (Table 4). 
Table 4. Independent samples t-tests between socio-demographic and problematic motor coordination ability.

\begin{tabular}{|c|c|c|c|c|c|c|c|c|}
\hline & $\begin{array}{l}\text { Control during movement } \\
\text { Mean } \pm \text { SD }\end{array}$ & $\begin{array}{l}\mathrm{T} \\
\text { value }\end{array}$ & $\begin{array}{l}\text { Fine motor/ } \\
\text { HandwritingMean } \pm \mathrm{SD}\end{array}$ & $\begin{array}{l}\mathrm{T} \\
\text { value }\end{array}$ & $\begin{array}{l}\text { General coordination } \\
\text { Mean } \pm \mathrm{SD}\end{array}$ & $\begin{array}{l}\mathrm{T} \\
\text { value }\end{array}$ & $\begin{array}{l}\text { Total score } \\
\text { Mean } \pm \text { SD }\end{array}$ & $\begin{array}{l}\mathrm{T} \\
\text { value }\end{array}$ \\
\hline Total sample & $26.09 \pm 3.7$ & & $17.3 \pm 2.7$ & & $21.24 \pm 3.4$ & & $64.64 \pm 8.6$ & \\
\hline \multicolumn{9}{|l|}{ Sex } \\
\hline Boys & $26.03 \pm 3.9$ & 0.329 & $16.67 \pm 2.9$ & $4.824^{\mathrm{c}}$ & $20.73 \pm 3.6$ & $3.067^{\mathrm{b}}$ & $63.43 \pm 9.3$ & $2.853^{\mathrm{b}}$ \\
\hline Girls & $26.15 \pm 3.6$ & & $17.87 \pm 2.3$ & & $21.70 \pm 3.1$ & & $65.72 \pm 7.8$ & \\
\hline \multicolumn{9}{|l|}{ Age } \\
\hline $6-7$ years & $25.30 \pm 3.9$ & $4.900^{\mathrm{b}}$ & $16.75 \pm 2.8$ & $4.576^{\mathrm{a}}$ & $20.64 \pm 3.6$ & $3.373^{\mathrm{a}}$ & $62.69 \pm 8.8$ & $5.487^{\mathrm{b}}$ \\
\hline $8-9$ years & $26.57 \pm 3.4$ & & $17.64 \pm 2.5$ & & $21.42 \pm 3.1$ & & $65.62 \pm 7.7$ & \\
\hline 10 and more years & $26.34 \pm 3.8$ & & $17.49 \pm 2.7$ & & $21.60 \pm 3.5$ & & $65.43 \pm 8.9$ & \\
\hline \multicolumn{9}{|l|}{ Gestational age at birth } \\
\hline Preterm & $25.88 \pm 3.9$ & 0.911 & $17.41 \pm 2.7$ & 0.030 & $21.06 \pm 3.7$ & 0.736 & $64.34 \pm 9.2$ & 0.699 \\
\hline Full term & $26.33 \pm 3.6$ & & $17.42 \pm 2.7$ & & $21.40 \pm 3.3$ & & $65.14 \pm 8.2$ & \\
\hline \multicolumn{9}{|l|}{ Out-of-school physical activity } \\
\hline$<3 \mathrm{~h} /$ week & $25.41 \pm 3.9$ & $4.373^{\mathrm{c}}$ & $16.93 \pm 2.7$ & $3.365^{\mathrm{c}}$ & $20.55 \pm 3.5$ & $4.953^{\mathrm{c}}$ & $62.89 \pm 8.7^{\mathrm{c}}$ & $4.971^{\mathrm{c}}$ \\
\hline$\geq 3 \mathrm{~h} /$ week & $26.90 \pm 3.4$ & & $17.77 \pm 2.5$ & & $22.08 \pm 3.0$ & & $66.75 \pm 7.8$ & \\
\hline \multicolumn{9}{|l|}{ Father educational level } \\
\hline First or second level studies & $25.44 \pm 3.9$ & $2.651^{\mathrm{b}}$ & $16.69 \pm 2.8$ & $3.104^{\mathrm{b}}$ & $20.67 \pm 3.6$ & $2.311^{\mathrm{a}}$ & $62.79 \pm 8.9$ & $3.040^{\mathrm{b}}$ \\
\hline Third level studies & $26.47 \pm 3.6$ & & $17.58 \pm 2.7$ & & $21.50 \pm 3.4$ & & $65.55 \pm 8.4$ & \\
\hline \multicolumn{9}{|l|}{ Mather educational level } \\
\hline First or second level studies & $24.80 \pm 4.0$ & $3.847^{\mathrm{c}}$ & $16.57 \pm 2.7$ & $2.797^{\mathrm{b}}$ & $20.64 \pm 3.2$ & 1.871 & $62.01 \pm 8.4$ & $3.301^{\mathrm{c}}$ \\
\hline Third level studies & $26.48 \pm 3.5$ & & $17.47 \pm 2.7$ & & $21.40 \pm 3.4$ & & $65.34 \pm 8.4$ & \\
\hline \multicolumn{9}{|l|}{ Family educational level } \\
\hline First or second level studies & $24.57 \pm 4.0$ & $3.804^{\mathrm{c}}$ & $16.36 \pm 2.8$ & $3.166^{\mathrm{b}}$ & $20.32 \pm 3.2$ & $2.526^{\mathrm{a}}$ & $61.25 \pm 8.6$ & $3.667^{\mathrm{c}}$ \\
\hline Third level studies & $26.39 \pm 3.6$ & & $17.47 \pm 2.7$ & & $21.43 \pm 3.4$ & & $65.29 \pm 8.4$ & \\
\hline \multicolumn{9}{|l|}{ Father occupational level } \\
\hline $\begin{array}{l}\text { Occupation not corresponding to university } \\
\text { studies }\end{array}$ & $25.86 \pm 3.7$ & $3.199^{\mathrm{c}}$ & $17.23 \pm 2.8$ & 0.979 & $21.02 \pm 3.5$ & $2.423^{\mathrm{a}}$ & $64.10 \pm 8.8$ & $2.630^{b}$ \\
\hline Occupation corresponding to university & $27.14 \pm 3.1$ & & $17.52 \pm 2.6$ & & $21.94 \pm 3.1$ & & $66.60 \pm 7.7$ & \\
\hline
\end{tabular}


Table 4. Independent samples t-tests between socio-demographic and problematic motor coordination ability.

\begin{tabular}{|c|c|c|c|c|c|c|c|c|}
\hline & $\begin{array}{l}\text { Control during movement } \\
\text { Mean } \pm \mathrm{SD}\end{array}$ & $\begin{array}{l}\mathrm{T} \\
\text { value }\end{array}$ & $\begin{array}{l}\text { Fine motor/ } \\
\text { HandwritingMean } \pm \text { SD }\end{array}$ & $\begin{array}{l}\mathrm{T} \\
\text { value }\end{array}$ & $\begin{array}{l}\text { General coordination } \\
\text { Mean } \pm \mathrm{SD}\end{array}$ & $\begin{array}{l}\mathrm{T} \\
\text { value }\end{array}$ & $\begin{array}{l}\text { Total score } \\
\text { Mean } \pm \text { SD }\end{array}$ & $\begin{array}{l}\mathrm{T} \\
\text { value }\end{array}$ \\
\hline \multicolumn{9}{|l|}{ studies } \\
\hline \multicolumn{9}{|l|}{ Mother occupational level } \\
\hline $\begin{array}{l}\text { Occupation not corresponding to university } \\
\text { studies }\end{array}$ & $26.03 \pm 3.7$ & 1.202 & $17.22 \pm 2.7$ & 1.013 & $21.08 \pm 3.5$ & 1.610 & $64.33 \pm 8.5$ & 1.486 \\
\hline $\begin{array}{l}\text { Occupation corresponding to university } \\
\text { studies }\end{array}$ & $26.50 \pm 3.7$ & & $17.51 \pm 2.6$ & & $21.66 \pm 3.3$ & & $65.67 \pm 8.5$ & \\
\hline \multicolumn{9}{|l|}{ Family occupational level } \\
\hline $\begin{array}{l}\text { Occupation not corresponding to university } \\
\text { studies }\end{array}$ & $25.79 \pm 3.7$ & $2.680^{\mathrm{b}}$ & $17.22 \pm 2.7$ & 0.929 & $20.98 \pm 3.5$ & $2.288^{\mathrm{a}}$ & $64.00 \pm 8.6$ & $2.367^{\mathrm{a}}$ \\
\hline $\begin{array}{l}\text { Occupation corresponding to university } \\
\text { studies }\end{array}$ & $26.76 \pm 3.5$ & & $17.47 \pm 2.6$ & & $21.74 \pm 3.2$ & & $65.97 \pm 8.2$ & \\
\hline
\end{tabular}

${ }^{\mathrm{a}} \mathrm{p}<0.05$.

${ }^{\mathrm{b}} \mathrm{p}<0.01$

$\mathrm{p}<0.001$. 
Thus, the outcomes of the linear regression analyses showed that being female, age, engaging in outof-school physical activity and family educational level were associated with fewer problematic levels of motor coordination ability (Table 5).

Table 5. Linear multivariate analysis to identify socio-demographic factors associated with motor coordination factors.

\begin{tabular}{|c|c|c|c|c|c|c|c|c|}
\hline \multirow{2}{*}{ Variable } & \multicolumn{2}{|c|}{$\begin{array}{l}\text { Control during } \\
\text { movement }\end{array}$} & \multicolumn{2}{|c|}{$\begin{array}{l}\text { Fine } \\
\text { motor/Handwriting }\end{array}$} & \multicolumn{2}{|c|}{$\begin{array}{l}\text { General } \\
\text { coordination }\end{array}$} & \multicolumn{2}{|c|}{ Total score } \\
\hline & B & $95 \% \mathrm{CI}$ & B & $95 \% \mathrm{CI}$ & B & $95 \% \mathrm{CI}$ & B & $95 \% \mathrm{CI}$ \\
\hline Sex (boys) & -0.140 & $-0.8-0.6$ & $-1.185^{\mathrm{c}}$ & $-1.7-(-0.7)$ & $-0.916^{\mathrm{b}}$ & $-1.6-(-0.3)$ & $-2.241^{\mathrm{b}}$ & $-3.8-(-0.6)$ \\
\hline \multicolumn{9}{|l|}{ Age } \\
\hline $6-7$ years & 0 & & 0 & & 0 & & 0 & \\
\hline 8 y 9 years & $1.512^{\mathrm{c}}$ & $0.7-2.4$ & $0.954^{\mathrm{b}}$ & $0.3-1.6$ & 0.747 & $0.0-1.5$ & $3.214^{\mathrm{c}}$ & $1.3-5.2$ \\
\hline 10 and more years & $1.090^{\mathrm{a}}$ & $0.2-2.0$ & $0.916^{\mathrm{b}}$ & $0.3-1.6$ & $0.928^{\mathrm{a}}$ & $0.1-1.7$ & $2.934^{\mathrm{b}}$ & $0.9-4.9$ \\
\hline Preterm birth (<37 weeks) & -0.316 & $-1.3-0.6$ & -0.079 & $-0.8-0.6$ & -0.329 & $-1.2-0.6$ & -0.724 & $-2.9-1.5$ \\
\hline Out-of-school physical activity ( $<3 \mathrm{~h} /$ week) & $-1.194^{\mathrm{c}}$ & $-1.9-(-0.5)$ & $-0.790^{\mathrm{b}}$ & $-1.3-(-0.3)$ & $-1.437^{\mathrm{c}}$ & $-2.1-(-0.8)$ & $-3.420^{\mathrm{c}}$ & $-5.0-(-1.8)$ \\
\hline $\begin{array}{l}\text { Family educational level (first or second level } \\
\text { studies) }\end{array}$ & $-1.101^{\mathrm{a}}$ & $-2.2-0.0$ & $-0.950^{\mathrm{a}}$ & $-1.7-(-0.2)$ & -0.538 & $-1.5-0.4$ & $-2.589^{\mathrm{a}}$ & $-5.0-(-0.2)$ \\
\hline $\begin{array}{l}\text { Family occupational level (occupation not } \\
\text { corresponding to university studies) }\end{array}$ & -0.501 & $-1.3-0.3$ & -0.028 & $-0.6-0.5$ & -0.515 & $-1.2-0.2$ & -1.044 & $-2.8-0.7$ \\
\hline
\end{tabular}

$B=B$ coefficient value CI $=95 \%$ confidence interval.

${ }^{\mathrm{a}} \mathrm{p}<0.05$.

${ }^{\mathrm{b}} \mathrm{p}<0.01$

${ }^{\mathrm{c}} \mathrm{p}<0.001$

\section{Discussion}

This study aimed to determine the prevalence of suspected DCD and its associations with sociodemographic factors in Spanish schoolchildren. To our knowledge, this is the first study to assess the prevalence of suspected DCD in a large sample of school-aged children in Spain. In this study, 12.2\% of children attending mainstream schools were identified as having suspected DCD and none of children had a previous clinical diagnosis of DCD according to parent reports. These findings are in line with other studies that report a similar rate of DCD. In Europe, the indication of DCD prevalence ranges from $4.9 \%$ in the United Kingdom to $19 \%$ in Greece, with similar outcomes in other regions such as Canada and South Africa (De Milander et al., 2016; Lingam et al., 2009; Tsiotra et al., 2006). However, it is noted that DCD prevalence rates may differ depending on the assessment used to establish DCD diagnosis. The studies that defined DCD cases using only one of the criteria derived from the DSM-IV or DSM-5 usually found a higher prevalence of DCD. For instance, Tsiotra et al. (2006) found that $8 \%$ of Canadian children and $19 \%$ of Greek children met DSM-5 criterion A of a DCD diagnosis that assessed motor competence, but did not evaluate the impact of motor coordination difficulties on everyday performance (i.e., criterion B). Using the MABC-2, $17.4 \%$ of Spanish pre-schoolers and $12 \%$ of South African grade 1 children were defined as having either DCD or at risk for DCD, but their functional performance was not evaluated, as only criterion A was considered (Amador-Ruiz et al., 2018; De Milander et al., 2016). The studies that have defined DCD primarily using criterion B (everyday functional impairment due to motor coordination difficulties), have also found a higher prevalence of DCD or suspected DCD. In Brazil, several studies using the DCDQ reported that approximately $30 \%$ of Brazilian children were classified as having DCD (Cavalcante Neto, Sato, \& Tudella, 2018; Della Barba, Luiz, Pinheiro, \& Lourenço, 2017). By contrast, Lingam et al. (2009), conducted a study with a large cohort of 7 and 8 year old children in the United 
Kingdom, assessing all DSM-IV criteria for DCD diagnosis, and found a significantly lower DCD prevalence, where only $1.8 \%$ of the children were diagnosed with DCD. It is important to consider all DSM-5 criteria to establish a DCD diagnosis, but this is often difficult when conducting large, population-based studies. While questionnaires and motor coordination test battery alone can be useful in identifying potential motor coordination problems, a definite diagnosis of DCD can only be established by considering all criteria (Blank et al., 2012). A recent study conducted in northwest Spain showed that only $1.09 \%$ of children received a clinical diagnosis of a neurodevelopmental motor disorder (Carballal Mariño et al., 2018). Given the high prevalence of suspected DCD in our study, in line with the findings of Amador-Ruiz et al. (2018), this fact suggests that in Spain, DCD is a hidden, unknown and underdiagnosed disorder.

Boys showed a higher prevalence of suspected DCD and higher frequency of problematic levels of motor coordination ability than girls, which has been reported by other studies and may be an indicator that boys are more likely to present motor coordination difficulties during early development (Beltrame et al., 2017; Delgado-Lobete \& Montes-Montes, 2017; Faebo Larsen et al., 2013). The underlying causes are not clear, though it has been suggested that differences in neurobiological and cultural factors between boys and girls may affect the development and quality of motor coordination (Blank et al., 2012; Rivard et al., 2014). It has been noted that preterm boys show increased adverse neurological outcomes than preterm girls, which could be a reason for the greater prevalence of DCD and other pervasive developmental disorders in boys (Fombonne, 2009; Zwicker, Missiuna, Harris \& Boyd, 2012). Regarding cultural factors, girls are often encouraged to engage in fine motor activities and therefore score higher in fine motor assessment tasks and tend to show a better motor coordination performance during early development (Amador-Ruiz et al., 2018; Delgado-Lobete \& Montes-Montes, 2017). Conversely, some studies have not found differences in DCD prevalence between boys and girls (Amador-Ruiz et al., 2018; Lingam et al., 2009; Silva \& Beltrame, 2013). This fact could be attributed to methodological differences, such as the selection of the assessment tools or the age of the children. Boys tend to score higher than girls in gross motor or aiming and catching tasks included in most of the motor coordination assessment batteries used in those studies and as such, boys are less likely to be identified as having DCD (AmadorRuiz et al., 2018; Lingam et al., 2009).

Older children showed a higher prevalence of suspected DCD but scored significantly higher in all motor coordination factors. As noted in previous research, children aged eight and older are more likely to present DCD symptoms, while simultaneously scoring better on the DCDQ (Cavalcante Neto et al., 2018; Faebo Larsen et al., 2013; Valentini et al., 2015). This could be because motor skills improve with age, and therefore, the DCDQ scores increase. However, those children with poor motor skills are more likely to be identified by the DCDQ as having suspected DCD. It has been noted that DCD diagnosis at early ages lacks stability (Blank et al., 2012). Additionally, DCD is a chronic condition that affects everyday performance and academic achievement, even during adolescence and adulthood (Harrowell, Hollén, Lingam, \& Emond, 2018), therefore it is possible that DCD becomes more evident and readily detected in older children because their motor coordination difficulties have a greater impact on their everyday activities.

In line with previous research, low gestational age at birth was associated with suspected DCD prevalence (Faebo Larsen et al., 2013; Kieviet et al., 2009; Lingam et al., 2009). An immature central nervous system can severely affect psychomotor development, which may explain why DCD is significantly more prevalent in preterm children, particularly among younger children. Low birth weight and gestational age at birth have both been shown to affect cognitive and behavioural outcomes, which may contribute to motor coordination difficulties in preterm children (Kieviet et al., 2009). In our study, low gestational age at birth was not statistically associated with suspected DCD according to logistic regression analyses, although preterm children were two times more likely to show DCD symptoms. This could be attributed to the age of the sample population, which included children older than eight years old. A meta-analysis conducted by Kieviet et al. (2009) indicated that the effect of gestational age on motor development may decrease as age increases, so older, preterm children and adolescents could show similar DCD prevalence than their term-born peers. 
Children with low participation in out-of-school physical activities were more than twice as likely to present DCD symptoms and problematic motor coordination ability skills. Children identified as having DCD tend to engage in less physical activities and sports than their typically developing peers, and usually show lower self-perception about their motor competence (Batey et al., 2014; Cermak et al., 2015; Fong et al., 2011; Magalhães et al., 2011). Limited physical activity participation reduces the number of opportunities to practice and improve motor skills, which may contribute to the development of DCD. Therefore, these children will engage less in social play and sports that require the fundamental movement skills they lack, and this process is likely to become a pattern that perpetuates itself (Schoemaker \& Smits-Engelsman, 2015). As low physical activity has been associated with low bone mineralization in children with DCD, this should be a major concern when approaching this disorder (Fong et al., 2018). Children with DCD show higher clinical obesity and low cardiorespiratory fitness prevalence than children without DCD (Tsiotra et al., 2006). Bone mineralization loss in obese, DCD children could contribute to the development of future clinical conditions.

Finally, low family educational level was also a factor associated with suspected DCD and problematic motor coordination skills. While previous research has demonstrated that family socioeconomic status is associated with DCD, studies usually focus solely on family economic level (Barba et al., 2017; Faebo Larsen et al., 2013; Lingam et al., 2009; Santos et al., 2015; Valentini et al., 2015). In our study, family educational level predicted DCD and motor coordination regardless of the occupational level of the family. As previously noted, children could be more likely to develop DCD if their parents have decreased recognition of motor coordination disability and access to health care (Lingam et al., 2009). Parents with a high educational level may be more conscious of the importance of psychomotor development or could be more likely to detect motor coordination difficulties, and thus could provide more opportunities of stimulation or have more access to health care services.

Several limitations of this study should be considered. First, although we used an established measure of daily living related motor skills, we could not establish a definite diagnosis of DCD since criterion A was not fully assessed and the DCDQ is not recommended for population screening (Blank et al., 2012). While motor coordination ability should be assessed using objective tests, parental reports can be valid instruments to assess motor coordination during everyday performance if the items are cross-culturally adapted and allow parents to compare motor skills within the same age groups (Blank et al., 2012; Zysset et al., 2018). As we aimed to evaluate a large sample size, the use of a motor coordination test battery was not feasible, and we chose to use a well-validated and cross-culturally adapted questionnaire to identify those children with suspected DCD. A second limitation is that we used a parent report of developmental disorders or learning difficulties without gathering clinical documentation. Only one child was reported as having a clinical diagnosis of developmental disorder (ADHD). While the sample came from mainstream schools, this may suggest that developmental disorders, and more precisely motor coordination disorders, are often underdiagnosed in Spain (Carballal Mariño et al., 2018). Socio-demographic factors associated with suspected DCD in our sample cannot be counted as risk factors due to the cross-sectional nature of our study, although this is valuable information that can be used in future studies. Finally, there may be a potential bias with the sample. It is possible that parent participation was influenced by their children's own motor performance or socioeconomic status. The sample comes from one city in Spain, and thus there may be differences when inferring to other Spanish regions. Parents were randomly selected and came from different socioeconomic districts and from both public and private schools to try to prevent this bias. 


\subsection{Implications for Spanish and international classrooms}

This study has important implications for Spanish and international classrooms. Our findings show that at least three children in a mainstream, Spanish classroom are at risk for developing DCD. This disorder is often hidden and underdiagnosed, not only in Spain, but in other regions, and yet the largest proportion of children that receive school-based occupational therapy is referred for handwriting difficulties (Missiuna et al., 2006, 2008). According to these findings, Spanish mainstream schools should consider including occupational therapists who would contribute to detecting and addressing occupational and scholastic limitations derived from motor coordination issues in children. Research has shown that task-oriented intervention methods are the most effective strategies to improve motor performance, but contextual barriers and socio-demographic factors need to be considered to design specific, effective programmes that accommodate the child's needs and characteristics (Blank et al., 2012). Schools in Spain and other regions could use these findings to address appropriate detection protocols that include those socio-demographic factors known to be associated with suspected DCD and to design strategies that allow children with suspected DCD to practice motor skills in an environment in which they can effectively engage in physical activities.

The DCDQ-ES scores provided here can be used by researchers in future studies and by teachers and health care practitioners in their daily classroom or clinical work. Researchers can also use the suspected DCD prevalence found in our study to warn national and international communities about this disorder. Future studies could explore the associations of additional socio-demographic and contextual factors, alongside those included in this study, to further investigate the external variables that may contribute to the development of DCD.

\section{Conclusions}

Suspected DCD affects approximately $12 \%$ of Spanish schoolchildren. Suspected DCD and problematic motor coordination ability skills are associated with age, sex, limited participation in out-ofschool physical activities, low gestational age at birth and family educational status. These are important findings as they point to the need to consider socio-demographics as relevant factors when assessing DCD in school-aged children. The impact of DCD on education achievement is not limited to childhood, but remains during adolescence (Harrowell et al., 2018), and thus it should be addressed as soon as motor coordination difficulties are displayed during development. Teachers and health care practitioners working in schools could use well-validated questionnaires to screen motor coordination difficulties, since they are easily accessed tools and useful for collecting information about the child's everyday performance. However, a DCD diagnosis should always include complementary assessments and the evaluation of all criteria. Schools offer the best environment for the early detection of motor coordination related learning difficulties and to develop strategies that promote motor coordination and minimize the impact of DCD on academic achievement. Occupational and physical therapists working in schools can help to detect and address this disorder using task-oriented intervention strategies that consider sociodemographic and contextual factors associated with DCD.

\section{Conflict of interest}

The authors declare that they have no conflict of interest.

\section{Funding sources}

This work was supported in part by a grant from the European Social Fund 2014-2020 and a predoctoral grant by Xunta de Galicia. 


\section{Acknowledges}

This work was supported in part by a grant from the European Social Fund 2014-2020 and a predoctoral grant by Xunta de Galicia. The authors would like to thank the families and schools that participated in this study and the teachers who assisted with data collection. The authors also thank the two anonymous reviewers for critically reading the manuscript and suggesting substantial improvements.

\section{References}

Amador-Ruiz, S., Gutierrez, D., Martínez-Vizcaíno, V., Gulias-González, R., Pardo-Guijarro, M. J., \& Sánchez-López, M. (2018). Motor competence levels and prevalence of developmental coordination disorder in Spanish children: The MOVI-KIDS study. The Journal of School Health, 88(7), 538-546.

American Psychiatry Association (2013). Diagnostic and statistical manual of mental disorders (fifth edition). Washington DC: American Psychiatry Association (DSM-5).

Barba, P. C. S. D., Luiz, E. M., Pinheiro, R. C., \& Lourenço, G. F. (2017). Prevalence of Developmental Coordination Disorder signs in children 5 to 14 years in São Carlos. Motricidade, 13(3),22-30.

Batey, C. A., Missiuna, C. A., Timmons, B. W., Hay, J. A., Faught, B. E., \& Cairney, J. (2014). Self-efficacy toward physical activity and the physical activity behavior of children with and without Developmental Coordination Disorder. Human Movement Science, 36, 258-271.

Beltrame, T. S., Capistrano, R., Alexandre, J. M., Lisboa, T., Andrade, R. D., \& Gomes Felden, E. P. (2017). Prevalência do Trastorno do Desenvolvimento da Coordenação en uma amostra de crianças brasileiras. Cadernos de Terapia Ocupacional, 25(1), 105-113.

Blank, R., Smits-Engelsman, B., Polatajko, H., \& Wilson, P. (2012). European Academy for Childhood Disability (EACD): Recommendations on the definition, diagnosis and intervention of developmental coordination disorder (long version). Developmental Medicine and Child Neurology, 54(1), 54-93.

Calahorro Cañada, F., Torres-Luque, G., López-Fernández, I., \& Álvarez Carnedo, E. (2014). Niveles de actividad física y acelerometría: Recomendaciones y patrones de movimiento en escolares. Cuadernos de Psicología del Deporte, 14(3), 129-140.

Carballal Mariño, M., Gago Ageitos, A., Ares Alvarez, J., Rio Garma, M., García Cendón, C., Goicoechea Castaño, A., et al. (2018). Prevalence of neurodevelopmental, behavioural and learning disorders in Pediatric Primary Care. Anales de Pediatría, 89(3), 153-161.

Cavalcante Neto, J. L., Sato, T. O., \& Tudella, E. (2018). Socio-demographic factors influences on guardians'perception of Developmental Coordination Disorder among Brazilian schoolchildren. Motriz: Revista de Educação Física, 24(2), e101810.

Cermak, S. A., Katz, N., Weintraub, N., Steinhart, S., Raz-Silbiger, S., Munoz, M., et al. (2015). Participation in physical activity, fitness, and risk for obesity in children with developmental coordination disorder: A cross-cultural study. Occupational Therapy International, 22(4), 163-173.

De Milander, M., Coetzee, F. F., \& Venter, A. (2016). Prevalence and effect of developmental coordination disorder on learning-related skills of South African grade one children. South African Journal for Research in Sport Physical Education and Recreation, 38(2), 49-62.

Delgado-Lobete, L., \& Montes-Montes, R. (2017). Psychomotor development and psychomotor profile of Spanish children between 3 and 6 years. Sportis Scientific Journal of School Sport Physical Education and Psychomotricity, 3(3), 454-470

Della Barba, P. C. S., Luiz, E. M., Pinheiro, R. C., \& Lourenço, G. F. (2017). Prevalence of Developmental Coordination Disorder signs in children 5 to 14 years in São Carlos. Motricidade, 13(3),22-30.

Domingo-Salvany, A., Regidor, E., Alonso, J., \& Alvarez-Dardet, C. (2000). Proposal for a social class measure. Working group of the Spanish society of epidemiology and the Spanish society of family and community medicine. Atención primaria, 25(5), 350-363.

Faebo Larsen, R., Hvas Mortensen, L., Martinussen, T., \& Nybo Andersen, A. M. (2013). Determinants of developmental coordination disorder in 7-year-old children: A study of children in the Danish National Birth Cohort. Developmental Medicine and Child Neurology, 55(11), 1016-1022.

Fombonne, E. (2009). Epidemioloy of pervasive developmental disorders. Pediatric Research, 66(6), 591598.

Fong, S. S., Lee, V. Y., Chan, N. N., Chan, R. S., Chak, W. K., \& Pang, M. Y. (2011). Motor ability and weight status are determinants of out-of-school activity participation for children with developmental coordination disorder. Research in Developmental Disabilities, 32(6), 2614-2623.

Fong, S. S. M., Vackova, D., Choi, A. W. M., Cheng, Y. T. Y., Yam, T. T. T., \& Guo, X. (2018). Diversity of activity participation determines bone mineral content in the lower limbs of pre-pubertal children with developmental coordination disorder. Osteoporosis International, 29(4), 917-925. 
Fuelscher, I., Caeyenberghs, K., Enticott, P. G., Williams, J., Lum, J., \& Hyde, C. (2018). Differential activation of brain areas in children with developmental coordination disorder during tasks of manual dexterity: An ALE meta-analysis. Neuroscience and Biobehavioral Reviews, 86,77-84.

Gomez, A., \& Sirigu, A. (2015). Developmental coordination disorder: Core sensori-motor deficits, neurobiology and etiology. Neuropsychologia, 79(Pt. B), 272-287.

Gomez, A., Piazza, M., Jobert, A., Dehaene-Lambertz, G., Dehaene, S., \& Huron, C. (2015). Mathematical difficulties in developmental coordination disorder: Symbolic and nonsymbolic number processing. Research in Developmental Disabilities, 43-44, 167-178.

Harrowell, I., Hollén, L., Lingam, R., \& Emond, A. (2018). The impact of developmental coordination disorder on educational achievement in secondary school. Research in Developmental Disabilities, $72,13-22$.

Haywood, K. M., Roberton, M. A., \& Getchell, N. (2012). Advance analysis of motor development. USA: Human Kinetics.

Huau, A., Velay, J. L., \& Jover, M. (2015). Graphomotor skills in children with developmental coordination disorder (DCD): Handwriting and learning a new letter. Human Movement Science, 42, 318-332.

Instituto Gallego de Estadística (2017). Información municipal y comarcal referente a la ciudad de A Coruña en 2017 (población según sexo y edad) [online data set].

Kieviet, J. F., Piek, J. P., Aarnoudse-Moens, C. S., \& Oosterlaan, J. (2009). Motor development in very preterm and very low-birth-weight children from birth to adolescence: A meta analysis. JAMA, 302(20), $2235-2242$.

Lingam, R., Hunt, L., Golding, J., Jongmans, M., \& Emond, A. (2009). Prevalence of developmental coordination disorder using the DSM-IV at 7 years of age: A UK population-based study. Pediatrics, 123(4), 693-700.

Magalhães, L. C., Cardoso, A. A., \& Missiuna, C. (2011). Activities and participation in children with developmental coordination disorder: A systematic review. Research in Developmental Disabilities, 32(4), 1309-1316.

Missiuna, C., Moll, S., King, G., King, S., \& Law, M. (2006). Missed and misunderstood: Children with developmental coordination disorder in the school system. International Journal of Special Education, 21,53-67.

Missiuna, C., Pollock, N., Egan, M., DeLaat, D., Gaines, R., \& Soucie, H. (2008). Enabling occupation through facilitating the diagnosis of developmental coordination disorder. Canadian Journal of Occupational Therapy, 75(1), 26-34.

Pons, R., \& Arufe, V. (2015). Análisis descriptivo de las sesiones e instalaciones de psicomotricidad de Educación infantil. Sportis Scientific Technical Journal, 2(1),125-146.

Prunty, M., Barnett, A. L., Wilmut, K., \& Plumb, M. (2016). Visual perceptual and handwriting skills in children with Developmental Coordination Disorder. Human Movement Science, 49,54-65.

Pulzi, W., \& Rodrigues, G. M. (2015). Developmental coordination disorder: A literature review. Revista Brasileira de Educação, 21(3), 433-444.

Rivard, L., Missiuna, C., McCauley, D., \& Cairney, J. (2014). Descriptive and factor analysis of the Developmental Coordination Disorder Questionnaire (DCDQ'07) in a population-based sample of children with and without Developmental Coordination Disorder. Child: Care, Health and Development, 40(1), 42-49.

Santos, V. A. P., \& Vieira, J. L. L. (2013). Prevalence of developmental coordination disorder in children aged 7 to 10 years. Revista Brasileira de Cineantropometria e Desempenho Humano, 15(2), 233-242.

Santos, V. A. P., Contreira, A. R., Caruzzo, N. M., Passos, P. C. B., \& Vieira, J. L. L. (2015). Developmental coordination disorder: An analysis of nutritional status and socioeconomic level. Motricidade, 11(1), $78-86$.

Schoemaker, M. M., \& Smits-Engelsman, B. C. M. (2015). Is treating motor problems in DCD just a matter of practice and more practice? Current Developmental Disorders Reports, 2(2), 150-156.

Shumway-Cook, A., \& Woollacott, M. H. (2010). Motor control: Translating research into clinical practice (3rd ed.). Philadelphia: Lippincott Williams \& Wilkins.

Silva, J., \& Beltrame, T. S. (2013). Indicative of developmental coordination disorder in students aged between 7 to 10 years. Revista Brasileira de Ciências do Esporte, 35(1), 3-14.

Tsiotra, G. D., Flouris, A. D., Koutedakis, Y., Faught, B. E., Nevill, A. M., Lane, A. M., et al. (2006). A comparison of developmental coordination disorder prevalence rates in Canadian and Greek children. Journal of Adolescent Health, 39(1), 125-127.

UNESCO Institute for Statistics (2012). International Standard Classification of Education (ISCED 2011). Canada: UNESCO Institute for Statistics.

Valentini, N. C., Clark, J. E., \& Whitall, J. (2015). Developmental co-ordination disorder in socially disadvantaged Brazilian children. Child: Care, Health and Development, 41(6), 970-979. 
Valentini, N. C., Coutinho, M. T. C., Pansera, S. M., Santos, V. A. P. D., Vieira, J. L. L., Ramalho, M. H., et al. (2012). Prevalence of motor déficits and developmental coordination disorders in children from South Brazil. Revista Paulista de Pediatria, 30(3), 377-384.

Wilson, B. N., Crawford, S. G., Green, D., Roberts, G., Aylott, A., \& Kaplan, B. J. (2009). Psychometric properties of the revised developmental coordination disorder questionnaire. Physical \& Occupational Therapy in Pediatrics, 29(2), 182-202.

Wilson, P. H., Smits-Engelsman, B., Caeyenberghs, K., Steenbergen, B., Sugden, D., Clark, J., et al. (2017). Cognitive and neuroimaging findings in developmental coordination disorder: New insights from a systematic review of recent research. Developmental Medicine and Child Neurology, 59(11), 11171129.

Zwicker, J. G., Missiuna, C., Harris, S. R., \& Boyd, L. A. (2012). Developmental coordination disorder: a review and update. European Journal of Paediatric Neurology, 16(6), 573-581.

Zysset, A. E., Kakebeeke, T. H., Messerli-Bürgy, N., Meyer, A. H., Stülb, K., Leeger-Aschmann, C. S., et al. (2018). The validity of parental reports on motor skills performance level in preschool children: A comparison with a standardized motor test. European Journal of Pediatrics, 177(5), 715-722. 